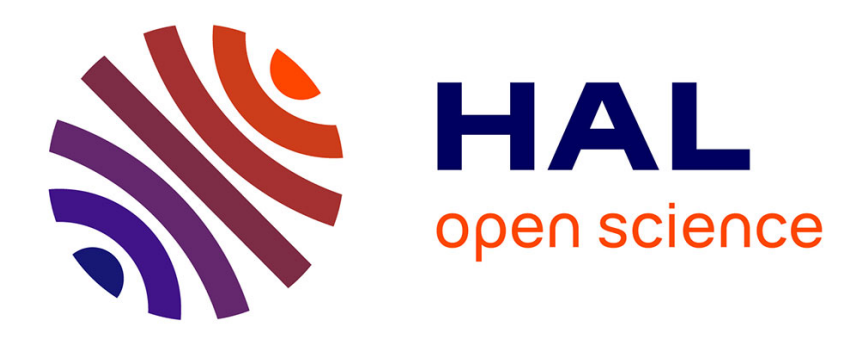

\title{
De l'impuissance à la toute-puissance: Proust et l'horreur de la pénétration
}

\author{
Isée Bernateau
}

\section{To cite this version:}

Isée Bernateau. De l'impuissance à la toute-puissance: Proust et l'horreur de la pénétration . Revue Française de Psychanalyse, 2012, Impuissance et frigidité 76 (1), pp.320. 10.3917/rfp.761.0027 . hal01496348

\section{HAL Id: hal-01496348 \\ https://hal.science/hal-01496348}

Submitted on 27 Mar 2017

HAL is a multi-disciplinary open access archive for the deposit and dissemination of scientific research documents, whether they are published or not. The documents may come from teaching and research institutions in France or abroad, or from public or private research centers.
L'archive ouverte pluridisciplinaire HAL, est destinée au dépôt et à la diffusion de documents scientifiques de niveau recherche, publiés ou non, émanant des établissements d'enseignement et de recherche français ou étrangers, des laboratoires publics ou privés. 


\section{De l'impuissance à la toute-puissance : Proust et l'horreur de la pénétration}

ISEE BERNATEAU

La sexualité de Marcel Proust, bien qu'il semble qu'elle ait été dans sa réalité, relativement pauvre et peu satisfaisante - au point que Jean-Yves Tadié note dans sa biographie : «Consolons-nous : nul historien n'a jamais classé les écrivains selon leurs performances sexuelles » (Tadié, 1996, p. 325) - a pourtant passionné les lecteurs et les critiques qui en ont traqué avec minutie les différentes manifestations. Sans doute cette fascination tient-elle à l'homosexualité, aux plaisirs clandestins et mystérieux auxquels elle est associée, ainsi qu'au sadisme et au masochisme qui s'y adjoignent ; sans doute aussi estelle renforcée par le matériau autobiographique dont les multiples travestissements et transpositions font qu'À la recherche du temps perdu apparaît comme un roman à clés dont le lecteur ne peut que se demander quel Albert se cache en réalité sous Albertine, même si Proust l'a averti en son temps de la vanité d'une telle entreprise. Mais, indépendamment de la réalité de la sexualité de l'homme Marcel Proust, dont on sait à la fois tout et rien, et dont il n'y a au fond rien à dire, comme toujours en ce qui concerne la sexualité d'un autre, s'il convient malgré tout de s'intéresser à la sexualité de l'écrivain Marcel Proust, c'est parce qu'il en a été le premier observateur voire le premier théoricien, et qu'il a lui-même scruté, dans toute son œuvre, et avec toute la «pénétration » qui fut sa marque de fabrique, son originalité, sa complexité, et même sa bizarrerie.

Car, si l'on regarde l'œuvre en effet, un certain nombre de traits se dessinent. Le plus saillant est qu'il s'agit d'une sexualité essentiellement masturbatoire, dont il semble - et sur ce point tous les commentateurs se rejoignent -, que la pénétration, active ou passive, ait été radicalement écartée. Si l'on s'en tient à la définition psychiatrique, Marcel Proust fut donc un impuissant primaire, « jamais parvenu au coït, ni avec une femme ni avec aucun autre partenaire sexuel »(Bourguignon, 1973, p. 21). De cette hantise de la pénétration, La Recherche témoigne à l'envie, multipliant les exemples d'un échec du narrateur à étreindre un objet qui toujours lui échappe. Parce qu'il ne peut jamais pénétrer l'objet de son désir, le narrateur se trouve réduit à devoir se contenter d'un effleurement superficiel : «D'autres jours, sa figure plus lisse engluait les désirs à sa surface vernie et les empêchait d'aller audelà » (Proust, 1918, p. 946). D’une sexualité qui se décline surtout en plaisirs de surface, caresses, baisers, accolements, le narrateur fait pourtant, par une ultime ruse de l'analogie, l'équivalent d'une pénétration : 
« Je revoyais Albertine s'asseyant à son pianola, rose sous ses cheveux noirs, je sentais mes lèvres qu'elle essayait d'écarter, sa langue maternelle, incomestible, nourricière, et sainte, dont la flamme et la rosée secrète faisaient que, même quand Albertine la faisait glisser à la surface de mon cou, de mon ventre, ces caresses superficielles mais en quelque sorte faites par l'intérieur de sa chair, extériorisée comme une étoffe qui montrerait sa doublure, prenaient, même par les attouchements les plus externes, comme la mystérieuse douceur d'une pénétration » (Proust, 1925, p. 73).

Description singulière, car la langue est ici à la fois l'organe externe érectile, sexe masculin qui en léchant la surface du corps le pénètre, ou tout du moins " essaie d'écarter » les lèvres du narrateur dans un geste évoquant le viol d'une femme, et l'organe interne concave qui accueille cette pénétration, sexe féminin « rose dans ses cheveux noirs » qui caresse par «l'intérieur de sa chair ». Le fantasme bisexuel annule la rencontre de deux sexes qui seraient différents et complémentaires au profit d'une totalité produisant un acte sexuel dans lequel l'intérieur et l'extérieur sont finalement réversibles. Or, cette annulation porte précisément sur la pénétration d'un sexe par l'autre, pénétration dont on pressent qu'elle véhicule un danger, au point que sa «mystérieuse douceur » évoquée par Proust résonne comme une dénégation venant contre-investir la menace qu'une telle langue pourrait faire peser sur le narrateur.

\section{Le baiser du soir, ou le contrat pervers mère-fils}

Mais si la langue d'Albertine se révèle ainsi dangereuse, c'est sans doute en effet d'être trop «maternelle », c'est-à-dire porteuse de cette fixation incestueuse à la mère qui la rend « incomestible » et « nourricière », « flamme » et « rosée », porteuse de contraires qui expliquent le caractère totalisant voire totalitaire des baisers qu'elle dispense. Le baiser d'Albertine rappelle sans cesse au narrateur un autre baiser, celui qui ouvre La Recherche, le «baiser maternel » donné ou refusé, viatique qui seul peut faire que la nuit ne soit pas la mort. À Combray, la scène initiatique du baiser du soir est une grande scène œedipienne qui se déroule en quatre tableaux. Premier tableau : la venue de M. Swann prive le narrateur du baiser de sa mère : «Mais le seul pour qui la venue de M. Swann devint l'objet d'une préoccupation douloureuse, ce fut moi. C'est que les soirs où des étrangers, ou seulement M. Swann, étaient là, maman ne montait pas dans ma chambre »(Proust, 1913, p. 23-38). Deuxième tableau : le narrateur, en proie à l'angoisse la plus extrême, voit « [son] esprit tendu par [sa] préoccupation » et tente d'attirer l'attention de sa mère par tous les moyens et notamment par une lettre. Exclu de « la fête inconcevable, infernale, au sein de laquelle nous croyions que des tourbillons ennemis, pervers et délicieux entraînaient loin de nous, la faisant rire de nous, celle que nous aimons », le narrateur espère par sa lettre y «pénétrer » : «Ces heures inaccessibles et suppliciantes où elle allait goûter des plaisirs inconnus, voici que par 
une brèche inespérée nous y pénétrons ». Le fantasme de scène primitive affleure dans les adjectifs qui qualifient la fête à laquelle la mère et ses invités prennent part et dont le narrateur est exclu, renvoyé à son ridicule de petit garçon. Il ne s'agit pas là d'une exclusion renvoyant à la détresse mais bien d'une exclusion dont le tranchant évoque la castration, une humiliation et une punition infligées pour ce fantasme lui-même.

Troisième tableau : parce que la mère n'a pas daigné répondre à sa lettre, le narrateur transgresse l'ordre d'aller se coucher et attend sa mère en haut des escaliers. L'angoisse de castration est de nouveau convoquée - espérée ? - quand la mère s'effraie de la transgression du fils et de la venue du père :

« Je vis dans la cage d'escalier la lumière projetée par la bougie de maman. Puis je la vis elle-même : je m'élançai. "Sauve-toi, sauve-toi, qu'au moins ton père ne t'ait pas vu ainsi attendant comme un fou !" Mais je lui répétais : "Viens me dire bonsoir", terrifié en voyant que le reflet de la bougie de mon père s'élevait déjà sur le mur, mais aussi usant de son approche comme d'un moyen de chantage et espérant que maman, pour éviter que mon père me trouvât encore là si elle continuait à refuser, allait me dire : "Rentre dans ta chambre, je vais venir." Il était trop tard, mon père était devant nous. Sans le vouloir, je murmurai ces mots que personne n'entendit: “Je suis perdu !" »

Rien ne manque à cette scène œdipienne emblématique, pas même la lumière sépulcrale des chandeliers rappelant le château de Combourg et le père terrifiant de Chateaubriand. Et pourtant, comme Proust l'écrit : «Il n'en fut pas ainsi »!

Il n'en fut pas ainsi car, dans le quatrième tableau, la phrase du père : " Mais va donc dormir avec lui, puisque tu disais justement que tu n'as pas envie de dormir, reste un peu dans sa chambre, moi je n'ai besoin de rien » met fin à la situation triangulaire de l'Edipe et enferme le narrateur dans une dyade mère-fils qui le plonge immédiatement et pour toujours dans la détresse: «Mais depuis peu de temps, je recommence à percevoir, si je prête l'oreille, les sanglots que j'eus la force de contenir devant mon père et qui n'éclatèrent que quand je me retrouvai seul avec maman. En réalité, ils n'ont jamais cessé. » À l'angoisse succède une immense tristesse. Le narrateur rapproche la silhouette de son père en haut de l'escalier d'une gravure dans laquelle Abraham, le père, intime à Sarah l'ordre de se séparer d'Isaac : « Il était encore devant nous, grand, dans sa robe de nuit blanche sous le cachemire de l'Inde violet et rose, qu'il nouait autour de sa tête quand il avait des névralgies, avec le geste d'Abraham dans la gravure d'après Benozzo Gozzoli que m'avait donné M. Swann disant à Sarah qu'elle a à se départir du côté d'Isaac. » La comparaison avec ce tableau est extrêmement curieuse, car c'est pourtant l'ordre exactement contraire qui est dans la scène du coucher à Combray proféré par le père. Mais le choix de cette gravure, la caractérisation féminine du père - en robe de nuit et en turban -, ainsi que la formule alambiquée, « qu'elle a à se départir du côté d'Isaac », 
résonnent autant de lapsus. Dans ces lapsus on discerne un désir à peine dicible de séparation affleurant sous la volonté proclamée de l'enfant de ne jamais quitter sa mère, mais on entend également l'échec de ce désir, échec qui renvoie définitivement le narrateur « du côté » de sa mère. Les étranges sanglots du narrateur, qui n'ont jamais cessé, témoignent du chagrin que procure la faillite du complexe adipien et l'instauration d'un contrat pervers mère-fils autorisé, voire même encouragé par le père.

Proust conclut : « Cette soirée commençait une ère, resterait comme une triste date. » Il y a bien dans cette scène une faillite qui n'échappe pas au narrateur. Dans Le Temps retrouvé, Proust revient sur cette scène inaugurale : «C'était de cette soirée, où ma mère avait abdiqué, que datait, avec la mort de ma grand-mère, le déclin de ma volonté, de ma santé » (Proust, 1927, p. 621). Green remarque que le père est comme « oublié » dans ce passage, oubli qui signale que, pour Proust, « le rapport préférentiel est le rapport mère/grand-mère » (Green, 2004, p. 102). Le renoncement du père à le faire renoncer à elle est donc bel et bien une abdication puisqu'il fait cesser le pouvoir souverain de l'interdit de l'inceste auquel le narrateur était jusque-là assujetti. Cet interdit avait auparavant dominé pour la mère, dans un pacte tacite qui la liait à son mari : « La concession qu'elle faisait à ma tristesse et à mon agitation en montant m'embrasser, en m'apportant ce baiser de paix, agaçait mon père qui trouvait ces rites absurdes, et elle eût voulu m'en faire perdre le besoin, l'habitude » (Proust, 1913, p. 13-38). Or, ce soir-là, la réaction du père fait passer le narrateur du statut d'enfant rebelle à celui, beaucoup plus délétère, d'enfant nerveux, un enfant dont l'attachement à la mère ne relève plus d'un désir interdit mais d'une maladie inguérissable : « Ainsi, pour la première fois, ma tristesse n'était plus considérée comme une faute punissable mais comme un mal involontaire qu'on venait de reconnaître officiellement, comme un état nerveux dont je n'étais pas responsable. » En abdiquant ainsi, la mère accepte qu'ils s'appartiennent pour toujours, ce qui a pour effet de tuer chez le narrateur toute volonté, toute créativité. Il faudra la lecture de François le Champi et le refoulé de toute sa thématique incestueuse - « quand c'était maman qui me lisait, à haute voix, elle passait toutes les scènes d'amour » - pour que les sanglots se calment et que le sommeil puisse advenir. Dans les épreuves du Contre SainteBeuve, le narrateur suit les méandres d'un de ses rêves et s'écrie : «Mais alors, si je suis dans cette chambre et convalescent, maman couche près de moi !» (Proust, 1908-1909, p. 60). Être malade, c'est donc pour Proust s'assurer de la pleine possession de maman, mais aussi ne pas pouvoir en jouir, puisqu'il est malade : la maladie est à la fois ce qui permet le rapproché incestueux et ce qui le punit.

C'est en tout cas au moment où il est absous que le narrateur se sent irrémédiablement coupable. Le caractère incestueux de son désir lui est comme dévoilé par la permissivité 
paternelle, et le rapproché avec la mère - «Maman passa cette nuit-là dans ma chambre » - a le parfum délétère de la profanation : «Certes, le beau visage de ma mère brillait encore de jeunesse ce soir-là où elle me tenait si doucement les mains et cherchait à arrêter mes larmes ; mais justement, il me semblait que cela n'aurait pas dû être, sa colère eût été moins triste pour moi que cette douceur nouvelle que n'avait pas connue mon enfance; il me semblait que je venais d'une main impie et secrète de tracer dans son âme une première ride et d'y faire apparaître un premier cheveu blanc. » Cette « trace » qu'il peut désormais inscrire sur le corps de la mère est une corruption, une pénétration sacrilège qui hâte son déclin et fait du fils un assassin : «Dans ces moments, rapprochant la mort de ma grand-mère de celle d'Albertine, il me semblait que ma vie était souillée d'un double assassinat » (Proust, 1925, p. 78). L'article «Sentiments filiaux d'un parricide ", que Proust fait paraître après qu'Henri Van Blarenberghe ait tué sa propre mère, est un véritable plaidoyer en faveur d'un meurtrier en qui Proust se reconnaît totalement : «Le pauvre parricide n'était pas une brute criminelle, un être en-dehors de l'humanité, mais un noble exemplaire d'humanité, un homme d'esprit éclairé, un fils tendre et pieux » (Proust, 1900-1908, p. 157).

\section{On profane une mère}

Le thème de la profanation de la mère, thème récurrent dans l'ensemble de l'œuvre de Proust, trouve donc dans la scène du coucher à Combray son origine romanesque. Ce thème prend dans les «profanations rituelles » de la scène de Montjouvain une représentation inversée, puisque le couple saphique y profane le portrait du père de mademoiselle Vinteuil sous les yeux voyeurs du narrateur (Proust, 1913, p. 157-163). À propos de Vinteuil, la mère du narrateur s'était écrié : «Pauvre Vinteuil, il a vécu et il est mort pour sa fille, sans avoir reçu son salaire. Le recevra-t-il et sous quelle forme ? Il ne pourrait lui venir que d'elle. » Cela avait paru énigmatique au narrateur, mais, après avoir vu le crachat sur le portrait du père et avoir constaté son efficace érotique sur mademoiselle Vinteuil, il se fait à lui-même cette réflexion : «Je savais maintenant, pour toutes les souffrances que pendant toute sa vie, $\mathrm{M}$. Vinteuil avait supportés à cause de sa fille, ce qu'après sa mort, il avait reçu d'elle en salaire. " Il est évident que c'est en grande partie son homosexualité qui apparaît à Proust comme une profanation de sa mère, une nouvelle qui pourrait la tuer. Mais on ne peut s'arrêter à ce seul niveau de compréhension. Les nouvelles de Proust, Violante ou la mondanité, ou La Confession d'une jeune fille, mettent en scène une jeune héroïne dont le premier orgasme vient tuer la mère. Dans ces nouvelles de jeunesse comme dans La Recherche, la profanation de la mère est l'ultime tentative du sujet pour créer un endroit de 
jouissance qui échappe à la mère, ainsi que le pied-de-nez qui signe et attaque le fait qu'il soit impossible de jouir sans elle. Dans ces nouvelles, la scène originaire s'inverse : le parent y assiste à une scène sexuelle entre enfants et, comme Michel Schneider le note, « regarde ce qui ne le regarde pas : la sexualité de son enfant »(Schneider, 1999, p. 374). Mais en convoquant le regard maternel comme adjuvant à sa jouissance, Proust la fait participer à ses ébats. Après avoir aidé Albert Le Cuziat à acheter l'hôtel Marigny pour en faire une maison de passe pour homosexuels, il y fait installer une partie du mobilier de sa mère. La transposition de cette anecdote dans La Recherche - « J'aurais fait violer une morte que je n'aurais pas souffert davantage » (Proust, 1918, p. 568) -, laisse apparaître le fantasme incestueux qui sous-tend l'acte sacrilège.

La faire participer, ou la tuer ? Le meurtre fait incontestablement partie des mises en scène sexuelles de Proust adulte, dont certains témoignages attestent qu'il aimait à observer les bouchers tuer les bêtes ou découper la viande - « Montre-moi comment on tue un veau » dit-il à un garçon boucher (Le Cuziat, 1985, p. 75). À l'hôtel Marigny, il était surnommé « l'homme aux rats ». Il demandait des rats qu'on piquait devant lui avec des épingles à chapeau. Le réseau métonymique des « rats », aussi complexe pour Proust que pour le célèbre homme aux rats freudien, renvoie à ses parents, à son père en particulier qui avait publié $L a$ Défense de l'Europe contre la peste, ainsi qu'à ses objets d'amour masculins - il appelait Lucien Daudet « Mon Rat ». Quand il ne pouvait parvenir à l'orgasme, « Albert apportait deux nasses. Dans chacune se trouvait un rat qui n'avait pas mangé depuis trois jours. On abouchait les nasses en soulevant les trappes qu'on avait disposées sur le lit. Aussitôt les deux bêtes affamées se jetaient l'une sur l'autre, en poussant des cris délirants et en se déchirant de leurs griffes et de leurs dents. Le plaisir de Marcel alors éclatait »(Jouhandeau, 1985, p. 80). Simulacre d'un coït sadique perpétré par les parents, d'une dévoration dont aucun des partenaires ne réchappera ? Proust relate un rêve dans lequel ses parents sont changés en souris blanches couvertes de pustules. Attaque haineuse contre la mère et fantasme de meurtre ? Le fait que Proust ait toujours sur lui la photographie de sa mère ou celle d'amies illustres et chères, évidents substituts maternels, et qu'il les exhibe devant un jeune garçon boucher, télégraphiste ou prostitué censé s'écrier : «Alors qu'est-ce que c'est c’te poule-là ?», irait dans ce sens. Le thème obsédant de la mère profanée y trouve sa source. Est à l'œuvre « la fixation incestueuse à la mère ou à la sœur » (Freud, 1912, p.130), fixation dont Proust se venge, et dont Freud fait la cause principale de l'impuissance. Est aussi à l'œuvre cette identification à la mère que Freud pointe dans le Léonard et qui fait de Proust un homosexuel qui aime «des personnes substitutives et des renouvellements de sa propre personne enfantine qu'il aime comme sa mère l'a aimé enfant » (Freud, 1910, p. 126), identification dont les 
relations du narrateur avec Albertine témoigne abondamment. Mais il y a néanmoins un « raté », qui tient sans doute, comme l'écrit Jean Cournut, au fait que : «l'identification à la mère est, elle-même, vécue comme incestueuse [...] et voilà qu'elle se trouve interdite à la réalisation homosexuelle comme n'importe quel vœu œdipien » (Cournut, 1998, p. 408).

Tuer la mère (en soi) pour pouvoir jouir, c'est donc pour Proust tenter de s'affranchir de cette identification qu'il vit comme un crime et qui lui interdit tout rapport et tout usage de son propre corps. À propos du baron de Charlus, personnage en qui il a déposé certains aspects, parmi les plus intimes et les plus inavouables, de lui-même, Proust se demande : « Au reste peut-on séparer entièrement l'aspect de $M$. de Charlus du fait que, les fils n'ayant pas toujours la ressemblance paternelle, même sans être invertis et en recherchant des femmes, il consomment dans leur visage la profanation de leur mère ? Mais laissons ici ce qui mériterait un chapitre à part : les mères profanées »(Proust, 1921, p. 300). Même si ce chapitre n'a jamais été écrit, on peut noter que la profanation au lieu même de la jouissance sexuelle attaque une imago maternelle anale toute-puissante et omniprésente dont le narrateur est devenu la propriété au moment de la défection du père. Dans Albertine disparue, il évoque « ce vieux désir de résistance à un complot imaginaire tramé contre moi par mes parents » (Proust, 1925, p. 230).

\section{Vivre sous emprise}

Roger Dorey repère dans la relation d'emprise perverse la volonté de la mère d' « exercer sur son enfant un contrôle omnipotent qui exige de la part de celui-ci une totale soumission [...] anéantissant toute possibilité de faire valoir un désir propre sauf à être la réplique exacte de celui qui le contraint »(Dorey, 1981, p. 122-123). À sa mère qui lui écrit en 1895 :

«Mon chéri,

Tes "dormi tant d'heures" continuent à ne me dire rien, ou plutôt rien qui vaille. Je demande et redemande :

Couché à

Levé à - - » (Proust, 1887-1905, p. 58). 
Proust répond la plupart du temps : «Rentré à onze heures vingt, même onze heures et quart, couché à minuit vingt, très peu d'oppressions, pris deux amyle préventivement, et en effet nuit sans oppression du tout » (Proust, 1887-1905, p. 78). Parfois, il tente de se révolter contre une relation dont il ne comprend que trop le marché qui la fonde, et qui pourrait se résumer à « ou bien tu existes par moi ou bien tu n'existes pas du tout ». Alors, la rage éclate : «La vérité c'est que dès que je vais bien, la vie qui me fait aller bien t'exaspérant, tu démolis tout jusqu'à ce que j'aille de nouveau mal. Ce n'est pas la première fois. J'ai pris froid ce soir ; si cela tourne en asthme qui ne saurait tarder dans l'état actuel des choses, je ne doute pas que tu seras de nouveau gentille pour moi quand je serai dans l'état où j'étais l'année dernière à pareille époque. Mais il est triste de ne pouvoir avoir à la fois affection et santé » (Proust, 1887-1905, p. 203-204). Ces tentatives de révolte contiennent en elles-mêmes leur abdication : mieux vaut en effet demeurer sous l'emprise d'une mère ambivalente, et s'en protéger par l'état de maladie, que risquer de perdre un amour dont Proust a le sentiment qu'il lui est si parcimonieusement accordé : «J'ai peur dans la violence de la crise qui m'empêchait d'écrire de n'avoir pas donné à mon mot la forme qui t'aurait plu. Car j'aime mieux avoir des crises et te plaire que te déplaire et n'en pas avoir » (Proust, 1887-1905, p. 233).

Pour se dégager de la mère anale, sans doute aurait-il fallu pouvoir «prendre appui sur une identification au Père porteur du phallus et par là, se soustraire à la maîtrise maternelle » (Torok, 1987, p. 169), ce qui, dans le cas de Proust achoppe sur le désintérêt paternel ainsi que sur la position de malade qui fait de lui à tout jamais l'objet d'emprise de la mère, du père et même du frère, lui aussi médecin. Les éventuels désirs de sortie de Proust hors du cercle familial se heurtent au discours incestueux de sa mère dont il est de fait le prisonnier avant que de devenir le geôlier d'Albertine. En 1897, suite à une querelle avec ses parents qui souhaitent qu'une photo représentant Lucien Daudet le contemplant amoureusement soit détruite, Proust les quitte tellement en colère qu'il brise en le frappant le vitrail de la porte de la salle à manger. Mais ce qui était tentative de séparation, voire de rupture de la part du fils est immédiatement inversé par le discours maternel qui, dans une lettre de réconciliation, fait de ce verre brisé le signe même de la fusion incestueuse : «Ne repensons plus et ne reparlons plus de cela. Le verre cassé ne sera plus que ce qu'il est au temple - le symbole de l'indissoluble union » (Proust, 1887-1905, p. 102). L'allusion séductrice au verre cassé lors du mariage rituel juif, en jouant sur l'implicite d'une complicité totale, unit le fils à la mère par les liens d'un mariage aussi secret qu'éternel. Le lien entre la mère et le fils est tel qu'il aboutit à une sorte de confusion des identités, rendant impossible de distinguer l'un de l'autre, consommant dans une identification réciproque un inceste psychique qui résonne comme un meurtre d'âme : «Peu à peu, ce fils dont elle avait voulu former l'intelligence, les mœurs, la 
vie, avait insinué en elle son intelligence, ses mœurs, sa vie même et avait altéré celles de sa mère » (Proust, 1971, p. 871). La porosité voire la confusion qu'une telle séduction opère se métaphorise dans La Recherche par la cloison qui sépare sa chambre de celle de sa grandmère, puis de sa mère, à Balbec. Cette cloison n'est pas tant une séparation qu'un point de réunion, elle est « une diaphanéité musicale » (Proust, 1921, p. 503), c'est-à-dire un voile qui ne cache rien et dont la transparence fait que ce qui s'y donne à entendre s'y donne aussi à voir.

En faisant de sa chambre, à la fin de sa vie, son seul espace vital, et en en interdisant soigneusement l'entrée, Proust a tenté de faire de cet espace un lieu inviolable. Au sein de cette réclusion, Céleste Albaret, la bonne très dévouée qui l'a soigné jusqu'à sa mort, joue un rôle-clé puisqu'elle devient l'esclave à même de lui fournir tout ce dont il pourrait avoir besoin sans jamais faire intrusion. L'identification à l'agresseur est patente, et Proust réalise une emprise obsessionnelle totale sur sa bonne, emprise dont le récit, consigné par Céleste Albaret, offre un témoignage aussi savoureux qu'effrayant. Dans cette relation de couple originale et platonique se réalise le fantasme d'un objet que Marcel peut sonner à tout moment mais qui reçoit en revanche comme ordre formel, et sous peine d'être congédiée, de ne le déranger sous aucun prétexte. S'appuyant sur la réalité de la domination sociale d'une domesticité encore totalement au service des maîtres, Proust met en scène une unité duelle avec une mère toujours à disposition, jamais manquante, mais qui ne peut plus exercer sur lui aucune emprise puisqu'il a désormais tout pouvoir sur elle. Ce qui était fantasme de possession absolue et d'enfermement dans La Prisonnière et dont Albertine disparue avait constaté l'échec cuisant est ainsi devenu, sous une forme quasi-parodiée, une réalité, les réduisant, lui et Céleste, à une claustration et une immobilisation totales. Sans doute cette ultime chorégraphie vient-elle incarner et sanctionner dans une jouissance à la fois masochiste et sadique le fantasme d'une emprise absolue sur un objet maternel séducteur aussi peu rassurant que contraignant.

\section{Noli me tangere}

Auparavant, la porosité entre le corps de la mère et celui du fils, et la mainmise qui s'y adjoignaient, avaient singulièrement privé Proust de toute pulsion d'emprise sur l'extérieur. Reclus dans sa chambre, il était condamné à observer ce qu'il renonçait à saisir : « Parfois, j'allais jusqu'à la fenêtre, je soulevais un coin du rideau. Dans une flaque d'or, suivies de leur institutrice, se rendant au catéchisme ou au cours, ayant épuré de leur souple démarche tout mouvement involontaire, je voyais passer de ces jeunes filles, pétries dans une chair 
précieuse, qui semblent faire partie d'une petite société impénétrable » (Proust, 1908-1909, p. 71). Face à des femmes « impénétrables », la seule position fantasmatique qu'il soit possible d'adopter est celle du voyeur contemplant une scène dans laquelle il ne peut jouer aucun rôle actif. Dans La Recherche, le narrateur se retrouve bien souvent à cette place, qu'il observe les ébats de mademoiselle Vinteuil et de son amie, ceux de Jupien et de Charlus, ou encore les rituels masochistes du baron de Charlus dans la chambre 43. Voyeur, le narrateur l'est aussi d'un baiser qu'il voit s'accomplir de manière idéale entre sa mère et sa grand-mère, alors qu'il échoue toujours, lui, à en ressentir le bénéfice : «Et penchée sur le lit, les jambes fléchissantes, à demi agenouillée, comme si, à force d'humilité, elle avait plus de chance de faire exaucer le don passionné d'elle-même, elle inclinait vers ma grand-mère toute sa vie dans son visage comme dans un ciboire qu'elle lui tendait »(Proust, 1921, p. 619-620). Marcel assiste là à un baiser sans retenue, une communion totale dont il n'est que le spectateur exclu. Amour réciproque et parfait, à l'image de la passion de Mme de Sévigné pour sa fille, amour dont les Lettres de Mme de Sévigné, que la grand-mère ne cesse de relire et qu'elle met au-dessus de tout, sont le bouleversant témoignage. S'il faut donc sans cesse et sans relâche au narrateur " posséder », voire enfermer l'objet de son amour, c'est parce qu'il est impossible de " pénétrer » à l'intérieur de cet amour sphérique entre mère et fille, amour dont les Lettres sont le blason et l'emblème. Comme Proust le lui fait remarquer dans une lettre, sa mère semble toujours se consacrer davantage à un autre qu'à lui : «Si je pouvais t'embrasser (sans te parler) cela me ferait tant de plaisir. Alors viens vite avant l'extinction des feux. Ne viens pas puisque hélas on a déjà sonné et que tu t'es précipité vers ma tante avec une ardeur que je ne t'ai jamais excitée » (Proust, 1187-1905, p. 232). Être le seul objet de l'amour maternel, voilà un vœu bien œdipien, mais qui achoppe sur une absence, un regard tourné vers une autre que lui.

\section{«Se faire casser le pot», ou les dangers de la jouissance génitale}

De même, dans La Recherche, la jalousie du narrateur, quand elle le confronte au fantasme des amours saphiques, se déchaîne sans limite parce qu'elle lui fait entrevoir « un fragment d'un autre monde, d'une planète inconnue et maudite, une vue de l'Enfer » (Proust, 1925, p. 99). Le plaisir féminin et son énigme se closent dans la mise en scène saphique d'un plaisir que l'homme, irréductible étranger au pays des femmes, est impuissant à donner. Plaisir qui exclut totalement la pénétration au profit de la caresse, du frôlement, comme l'affirme le docteur Cottard au narrateur : «Tenez, regardez, ajouta-t-il en me montrant Albertine et Andrée qui valsaient lentement, serrées l'une contre l'autre, j'ai oublié mon 
lorgnon et je ne vois pas bien, mais elles sont cependant au comble de la jouissance. On ne sait pas assez que c'est surtout par les seins que les femmes l'éprouvent. Et, voyez, les leurs se touchent complètement » (Proust, 1921, p. 498). Seins phalliques victorieux qui jouissent de se toucher, l'énigme de la sexualité féminine, énoncée dans la théorie fantaisiste du docteur Cottard, se résume à cette équation qui conjugue plaisir extrême et absence de phallus, loin des affres de l'angoisse de castration. À l'inverse, la scène de masturbation dans le cabinet sentant le lilas donne au narrateur un sentiment de danger pour sa vie même : « L'exploration que je fis alors en moi-même, à la recherche d'un plaisir que je ne connaissais pas, ne m'aurait pas donné plus d'émoi, plus d'effroi s'il s'était agi pour moi de pratiquer à même ma moelle et mon cerveau une opération chirurgicale. À tout moment je croyais que j'allais mourir »(Proust, 1921, p. 54-56). Le plaisir engendré entraîne néanmoins un sentiment d'élation et de puissance, mais la scène se clôt sur l'odeur du lilas, à laquelle « une odeur âcre, une odeur de sève s'y mêlait, comme si j'eusse cassé la branche ». La « branche cassée » du petit cabinet rencontre un écho singulier dans La Recherche quand Albertine réveille la fureur jalouse du narrateur en laissant échapper qu'elle aimerait mieux « se faire casser le pot». Étrange lapsus proustien, étrange expression pour désigner une sexualité lesbienne, cette « casse » semble bien plutôt menacer celui qui se laisserait pénétrer passivement par son partenaire, renouant avec le fantasme d'un coït destructeur faisant voler en éclats l'intégrité corporelle sous les coups de «la férocité de la part du corps qui jouit »(Proust, 1896, p. 95).

La description du corps d'Albertine, description qui a donné lieu à tant de commentaires en raison de son androgynie et de son ambiguïté, est exemplaire d'une difficulté à sexuer le corps, à se représenter aussi bien un corps de femme qu'un corps d'homme : «Ses deux petits seins haut remontés étaient vraiment si ronds qu'ils avaient moins l'air de faire partie intégrante de son corps que d'y avoir mûri comme deux fruits ; et son ventre (dissimulant la partie qui chez l'homme s'enlaidit d'un crampon resté fiché dans une statue descellée) se refermait, à la jonction des cuisses, par deux valves d'une courbe aussi assoupie, aussi reposante, aussi claustrale que celle de l'horizon quand le soleil a disparu » (Proust, 1922, p. 79). Dans le corps d'Albertine les deux sexes se condensent, créant un être bisexuel qui retient prisonnier un phallus non plus érigé mais au contraire en péril. L'aspiration bisexuelle retrouve l'angoisse de castration là où elle tente de s'en affranchir, rejoignant « la tête de Méduse » dans laquelle Freud voit une figuration de l'horreur de la castration, atténuée par les cheveux qui « remplacent le pénis dont le défaut est la cause de l'horreur » (Freud, 1922, p. 163).

Mais l'angoisse de castration est chez Proust débordée par une angoisse de néantisation dont la description du sexe d'Albertine porte la trace, et dont l'horreur de la pénétration porte 
la trace. À la vision angoissée d'un phallus qui décidément ne tient pas, objet partiel détachable greffé artificiellement sur le reste du corps et dont la chute est annoncée par le descellement de la statue, répond un corps féminin totalement clos et refermé sur lui-même, « claustral ». Deux « valves », coquilles close, obturent le sexe d'Albertine, et par extension celui de la mère, et, comme le note Michel Schneider : «La valve n'est pas une vulve. Maman n'est pas une femme » (Schneider, 1999, p. 175). La paronomase valve/vulve fonctionne comme un lapsus venant dénier le trou, l'ouverture, au profit de la fermeture hermétique d'une coquille-carapace protégeant un intérieur aussi inaccessible qu'inviolable. Le danger est celui d'une ouverture démesurée, voire infinie, du sexe féminin, ouverture qui prend dans Jean Santeuil des accents mythologiques. Petit enfant, alors que Jean voit sa mère se baigner dans la piscine Deligny, l'eau des bains «paraissant sans fond, il avait eu le sentiment que sans doute - comme les anciens croyaient qu'à un certain endroit non loin de Pouzolles était l'entrée des enfers - là était l'entrée des mers glaciales »(Proust, 1895, p. 305-306). Perdu dans la contemplation de ce monde terrifiant des mers infernales, il lui sembla qu'il était « le fils d'une déesse et qu'ainsi il avait pu voir l'entrée de ce monde fantastique inconnue de tous et pourtant si près du pont de la Concorde, près de laquelle tout le monde passait sans le savoir, de même que nous marchons tous les jours par-dessus d'immenses égouts navigables, dont l'entrée n'est pas visible : mais le préfet de police et d'autres font au milieu d'une place soulever une pierre qui ressemble à toutes les autres et y descendent ». Rien ne manque dans ce fantasme de pénétration : l'entrée du trou est secrète et invisible à tous ; seul le représentant de la loi, le préfet de police, « et d'autres », qui restent mystérieux, anonymes et possiblement inquiétants, ont le droit d'y pénétrer. Mais l'ouverture infinie du sexe maternel est remarquable, dans une démultiplication qui fait que l'eau des bains Deligny s'ouvre sur la Seine qui s'ouvre elle-même sur des mers allant jusqu'aux «pôles », laissant « un monde probablement parallèle à l'autre et en-dessous, et où on ne voyait pas la lumière du ciel ». Véritable Thalassa, ce continent noir ne peut que provoquer l'effroi du narrateur : les mers dans lesquelles il voit «sa mère s'y jouer en riant, lui envoyer des baisers » sont des mers « glaciales », mers des enfers à « la puissance irritée ». Mère froide donc, mère inquiétante, déesse au pouvoir illimité. C'est bien du pouvoir du sexe de la mère qu'il s'agit ici, pouvoir de vie, mais aussi de mort sur un fils qui le regarde fasciné, écrasé par une démesure qui déborde complètement la dissymétrie. 


\section{On suture une mère}

Face à un creux dont les contours sont aussi peu circonscrits, l'angoisse est moins celle d'une castration que d'un engloutissement, danger dont on comprend qu'il rende nécessaire de fermer la vulve par une valve hermétique. La Philosophie dans le boudoir de Sade offre de cette suturation du creux maternel une version aussi spectaculaire que littérale. L'éducation libertine d'Eugénie se clôt par une ultime cruauté destinée à éprouver la jeune élève et à permettre l'accomplissement du programme politique sadien de renversement de la morale au profit de la loi naturelle : Eugénie doit coudre « le con et le cul » de sa propre mère. La couture, suprême vengeance sadique, ferme un sexe dont le trou maternel crée un effroi qu'aucune jouissance ne peut endiguer. En faisant de La Recherche une « robe », infiniment reprisée, ravaudée, qui revêt le corps de la mère, Proust donne de cette suture une version plus métaphorisée. La robe de mots cache la nudité de la mère et détourne le regard de ce qu'on ne saurait y voir, mais elle souligne et circonscrit aussi les contours de son corps, permettant de donner une forme à l'informe $\mathrm{du}$ « trou ». Jean-Pierre Richard remarque que la conduite d'eau, métaphore phallique s'il en est, est un motif proustien récurrent, dont le bruit dans un calorifère procure au narrateur une extase mémorielle lors de son séjour à Doncières. Or, dans Jean Santeuil, texte qui préfigure et anticipe La Recherche, cette conduite d'eau est menacée d'éclater par le contact avec ce qui l'entoure : "Près d'un bassin d'où l'eau descendait alimenter des pompes et au fond duquel les tuyaux apparents et croisés avaient déjà cessé d'être une œuvre de l'homme ; et se mirant au fond des eaux qu'elle verdissait, la délicieuse graine verdâtre de mille invisibles mousses aquatiques les enveloppait, se mêlant, se nouant les unes aux autres parfois si fort qu'elles avaient failli les crever et à un endroit les avaient tout à fait infléchis » (Proust, 1895, p. 330). La reprise de ce motif, dans les dernières pages du Temps retrouvé, le transforme complètement, annulant le danger du contact du sexe avec ce qui l'enserre au profit d'une rencontre harmonieuse entre deux éléments enfin complémentaires : «Une simple relation mondaine, même un objet matériel, si je le retrouvais au bout de quelques années dans mon souvenir, je voyais que la vie n'avait pas cessé de tisser autour de lui des fils différents qui finissait par le feutrer de ce beau velours inimitable des années, pareil à celui qui dans les vieux parcs enveloppe une simple conduite d'eau d'un fourreau d'émeraude » (Proust, 1927, p. 604). De l'entrée des mers infernales immenses et glaciales au fourreau d'émeraude gainant le pénis de son beau velours, le parcours littéraire permet la représentation métaphorique d'un vagin enfin circonscrit. Seul le Temps, grand héros de la Recherche, par les fils qu'il tisse entre passé et présent, autorise le narrateur, dans et par la littérature, à étreindre l'ultime objet de son désir. 
C'est en effet seulement en devenant écrivain que Proust pût se permettre d'approcher, la divinité maternelle. L'épisode si célèbre de la madeleine - prénom de la mère-épouse de François le Champi et de Madeleine Lemaire, au nom évocateur, substitut amoureux et platonique de la figure maternelle - place le style proustien sous l'égide du principe de plaisir qui fait fi de la marche du temps et se rit de sa force de destruction : « Mais à l'instant même où la gorgée mêlée des miettes de gâteau toucha mon palais, je tressaillis, attentif à ce qui se passait d'extraordinaire en moi. Un plaisir délicieux m'avait envahi, isolé, sans la notion de sa cause » (Proust, 1913, p. 44-47). Faire pénétrer la mère en soi, l'avaler, la détruire et en jouir dans une oralité dyonisiaque, voilà un plaisir insolite qui ouvre les portes d'un monde nouveau, dans lequel la toute-puissance va de pair avec un sentiment d'immortalité : «J'avais cessé de me sentir médiocre, contingent, mortel. » Par le miracle d'une analogie, le temps s'abolit et le monde de l'enfance se déploie, tel qu'il était, " de ma tasse de thé ». L'animisme infantile, pensée magique qui résout la contradiction entre identité de perception et identité de pensée, est au cœur de l'écriture proustienne : la réalité recréée n'est pas seulement une réminiscence de la réalité passée, elle est le passé car elle est soutenue par la force de l'hallucinatoire. La force de la sexualité infantile anime et soutient un texte dont la jubilation se communique aux lecteurs qui font eux aussi, en lisant La Recherche, l'expérience singulière d'une plongée au cœur d'un monde dans lequel « la disparition du complexe d'Edipe » n'est pas au programme. En faisant de La Recherche, non pas seulement une robe mais aussi « une cathédrale » (Proust, 1925, p. 610) à la gloire et à la mémoire de sa mère morte, Proust réalise un monument dans lequel il peut pénétrer et se promener à sa guise, ce à quoi il s'est consacré pendant les dernières années de sa vie où son vagabondage dans les pages de son œuvre avait remplacé chez lui toute autre forme de périple. À l'image de ces « cathédrales mortes » auxquelles des « artistes, séduits par le rêve de rendre la vie à ces grands vaisseaux qui s'étaient tus, veulent en refaire pour une heure le théâtre du drame mystérieux qui s’y déroulait » (Proust, 1900-1908, p. 142), À la recherche du temps perdu permet un corps-à-corps sublimé avec une mère morte dont l'habit de mots borne, diffracte, et démultiplie la jouissance.

Isée Bernateau

3 rue Jean du Bellay

75004 Paris

REFERENCES BIBLIOGRAPHIQUES

Baudin A., Coblence F. (1999), Marcel Proust visiteur des psychanalystes, Paris, PUF. 
Bonnet H. (1985), Les Amours et la sexualité de Marcel Proust, Paris, Nizet.

Bourguignon A. et al., L’impuissance sexuelle psychique, Perspectives psychiatriques, 1973, 40, 1, 21-31.

Cournut J. (1998), Le pauvre homme, ou pourquoi les hommes ont peur des femmes ?, RFP, t. LXII, $\mathrm{n}^{\circ} 2$, p. $393-$ 405.

Dorey R. (1981), La relation d'emprise, Nouvelle revue de Psychanalyse, ${ }^{\circ}$ 24, p. 117-140.

Freud S. (1909), L’Homme aux rats, OCF.P, IX, Paris, PUF, 1998.

- (1910), Un souvenir d'enfance de Léonard de Vinci, OCF.P, X, Paris, PUF, 1993.

- (1912), Du rabaissement généralisé de la vie amoureuse », OCF.P, XI, Paris, PUF, 1998.

- (1940 c [1922]), La tête de Méduse, OCF.P, XVI, Paris, PUF, 2003.

Green A. (2004), La Lettre et la Mort, Paris, Denoël.

Proust M., Les Plaisirs et les jours (1896), Jean Santeuil (commencé en 1895, publié en 1952), Paris, Gallimard, «Bibliothèque de la Pléiade », 1971. (LPJ) (JS)

- Pastiches et mélanges (1900-1908), Contre Sainte-Beuve (1954), Essais et articles. Paris, Gallimard, «Bibliothèque de la Pléiade », 1971.

- (1913-1918), Du Côté de chez Swann, À l'Ombre des jeunes filles en fleurs, I, À la Recherche du Temps perdu, I, Paris, Gallimard, « Bibliothèque de la Pléiade », 1987.

- (1918-1921), À l’Ombre des jeunes filles en fleurs, II, Le Côté de Guermantes, À la Recherche du Temps perdu, II, Paris, Gallimard, « Bibliothèque de la Pléiade », 1988.

- (1921-1922), Sodome et Gomorrhe, La Prisonnière, À la Recherche du Temps perdu, III, Paris, Gallimard, «Bibliothèque de la Pléiade », 1988.

- (1925-1927), Albertine disparue, Le Temps retrouvé, À la Recherche du Temps perdu, IV, Paris, Gallimard, «Bibliothèque de la Pléiade », 1989.

- (1908-1909), Contre Sainte-Beuve, Paris, Gallimard, « Folio », 1954.

- (1887-1905), Correspondance avec sa mère, Paris, Plon, 1953.

Sade (1794), La Philosophie dans le boudoir, Paris, Gallimard, « Folio », 1976. 
Schneider M. (1999), Maman, Paris, Gallimard, « Folio ».

Tadié J.-Y. (1996), Marcel Proust, Paris, Gallimard, «Folio ». 
Titre article

Auteur ARticle

Texte de l'éventuelle citation en exergue

Signature exergue

Texte courant

INTERTITRE 1

Texte courant

Intertitre 2

Texte courant

Intertitre 3

Texte courant

Signature et coordonnées postales

BIBLIOGRAPHIE

Bibliographie texte

RESUMAUTEUR. - titre de l'article

Résumé - Résumé de l'article

Mots clés - mots clés de l'article 